\title{
Neurogenic dysphagia experiences/characteristics at a tertiary center-retrospective analysis
}

\author{
(1) Berke Aras ${ }^{1}$, (1) Özgü İnal' ${ }^{2}$, (1) Serdar Kesikburun ${ }^{3}$, (1) Rıdvan Alaca ${ }^{4}$, (1) Evren Yaşar $^{3}$ \\ ${ }^{1}$ Kastamonu Rehabilitation Center, Department of Physical Medicine and Rehabilitation, Kastamonu, Turkey \\ 2University of Health Sciences Turkey, Faculty of Gülhane Health Sciences, Department of Occupational Therapy, Ankara, Turkey \\ 3University of Health Sciences Turkey, Faculty of Gülhane Health Sciences, Department of Physical Medicine and Rehabilitation, \\ Ankara, Turkey
}

${ }^{4}$ Liv Hospital Ankara, Clinic of Physical Medicine and Rehabilitation, Ankara, Turkey

\section{Date submitted:}

26.02.2021

Date accepted:

07.11 .2021

Online publication date:

15.03.2022

\section{Corresponding Author:}

Berke Aras, M.D., Kastamonu

Rehabilitation Center, Department of

Physical Medicine and Rehabilitation,

Kastamonu, Turkey

drberkearas@gmail.com

ORCID:

orcid.org/0000-0002-2761-3478

Keywords: Brain injury, neurogenic dysphagia, stroke, vascular involvement, swallow therapy, rehabilitation

\begin{abstract}
Aims: To compare demographic and clinical features and their relation to rehabilitation outcomes in patients impaired swallowing after stroke or traumatic brain injury (TBI), anoxic brain injury, encephalitis and glioma.
\end{abstract}

Methods: This retrospective study evaluated the medical records of patients with brain injury in our university rehabilitation. Patients who received swallowing therapy for 24 sessions (3 days a week for 8 weeks) were included. Swallowing was evaluated with a functional oral intake scale (FOIS) and videofluoroscopic swallowing study before and after rehabilitation. Videofluoroscopy images were scored using the Penetration Aspiration Scale (PAS). Functional status was assessed with the Functional Independence Measure.

Results: The study included 271 patients [stroke: 175 (64.6\%); TBI: 58 (21.4\%); anoxic brain injury: 13 (4.8\%); glioma: 18 (6.6\%); and encephalitis 7 (2.6\%)]. Significant improvement was observed on the FOIS in stroke (pretreatment: $5.9 \pm 1.9$ vs. posttreatment: $6.1 \pm 1.9, p=0.011$ ) and TBI groups (pretreatment: $5.3 \pm 2.4$ vs. posttreatment: $5.9 \pm 7.0, p=0.007$ ). In both groups, significant improvements were observed in all three consistencies (solid, pudding, and liquid) according to PAS after treatment $(p<0.05)$. Stroke patients with the middle cerebral artery (MCA) and posterior cerebral artery syndromes showed statistically significant improvement on FOIS. Patients with the MCA syndrome also showed significant improvement in the liquid score of the PAS.

Conclusions: Swallowing therapy was found effective in improving swallowing functions in patients with stroke and TBI. In particular, stroke patients with MCA involvement gained more benefit from treatment.

\section{Introduction}

Swallowing is a complex sensorimotor event that involves both voluntary and involuntary processes. Structures at many levels from the cerebral cortex to the bulbus within the central nervous system are involved in swallowing (1). Dysphagia, which is the medical term for having difficulty swallowing, is common in patients with a number of neurological disorders. Dysphagia may even occur secondary to peripheral nervous system lesions, muscle disorders, and neuromuscular junction disorders (2).
Neurogenic dysphagia is usually caused by brain damage. It may lead to lethal complications such as aspiration, pneumonia, malnutrition, and dehydration (3). The incidence of post-stroke dysphagia is $30-50 \%$ (4). Approximately $27-61 \%$ of patients after traumatic brain injury (TBI) have swallowing disorders due to cognitive and behavioral problems, loss of neuromuscular control, intubation, and tracheostomy history $(5,6)$. Patients with a history of high-grade glioma or encephalitis may also have neurogenic dysphagia with progressive neurological and cognitive deficits. 
Before recommending any specific treatment, experienced physical medicine and rehabilitation specialists and swallowing therapists should locate and identify the mechanism underlying the swallowing problem with a comprehensive evaluation. Various tests can help diagnose swallowing problems. Videofluoroscopy is the gold-standard method in assessing swallowing disorders. Fiberoptic endoscopic examination, ultrasonography, scintigraphy, and pharyngeal manometry can also be used. Diet modifications, postural maintenance, compensatory strategies, swallowing maneuvers, exercises, electrical stimulation, botulinum toxin administration, or surgical methods are the most common treatment methods of neurogenic dysphagia (7).

Neurogenic dysphagia rehabilitation is of vital importance in patients with neurological diseases to provide proper nutrition and prevent secondary complications. However, there is no consensus on how treatment will be administered in which patient group. Although most interventional treatment studies have been conducted in patients with stroke, rehabilitation studies of patient groups such as TBI, anoxic brain injury, glioma, and encephalitis are still not sufficient. The present study documents the clinical functioning outcomes of patients with neurogenic dysphagia who have been treated in a tertiary rehabilitation hospital in Turkey. The aims of this study were 1) to compare rehabilitation outcomes in patients with stroke and other acquired brain injuries; 2) to determine the features of dysphagia according to the vascular area involvement in stroke.

\section{Methods}

\section{Study design and participants}

In this single-center, retrospective study, we reviewed the electronic medical records of the patients with acquired brain injury $(A B I)$ who were admitted to a university rehabilitation hospital between January 2010-December 2014. All cases between these dates were examined for inclusion in the study and patients who had a history of swallowing problems after brain damage was included. The study protocol was approved by the Local Ethics Committee (Gülhane Military Medical Academy, decision no: 1642-63250, date: 04.11.2014).

Demographic data including etiology of brain injury, age at the time of injury, sex, time since injury, history of aspiration pneumonia, and history of tracheostomy were collected. Arterial involvement in patients with stroke was determined using MRI findings.

\section{Clinical assessment}

Functional status was assessed with the Functional Independence Measure (FIM). The validity and reliability of the FIM for Turkish stroke survivors was performed by Küçükdeveci et al. (8). Patients were evaluated with a videofluoroscopic swallowing study and the functional oral intake scale (FOIS) at baseline and after completing the rehabilitation program. For the videofluoroscopic swallowing study, radiopaque was used as a contrast agent to visualize the bolus. Imaging shots were taken during the swallow. Swallowing functions were evaluated in three different consistencies as defined previously by Luchesi et al. (9) study: liquid, pudding, and solid. The pudding consistency was prepared according to the recommendation of the manufacturer of the food thickener used (Nestle thicken-up ${ }^{\circledR}$ ). Liquid and solid consistencies consisted of water and a standard cracker, respectively. While the patient was sitting in a wheelchair between the C-arm of the device (Ziehm Imaging, Vario DDD, Nürnberg, Germany) the patient's head was positioned on the midline, facing up. Images were adjusted to include the lips at the front, the cervical vertebra at the back, the soft palate at the top, and the C7 vertebra at the bottom. The presence of penetration-aspiration, degree of penetration-aspiration, and presence of pharyngeal residue were recorded.

Penetration Aspiration Scale (PAS) was used to determine the penetration-aspiration severity. The validity and reliability study of the PAS was conducted for the Turkish population (10). Obtained images from the video-fluoroscopy were scored using this scale. The presence of penetration-aspiration, degree of penetration-aspiration, and presence of pharyngeal residue were recorded. Material entering the airway and contacting the vocal folds was defined as penetration; material passing below the vocal folds was defined as aspiration. In the video-fluoroscopic swallowing study, aspiration is observed when the bolus passes through the glottis. Scores on the PAS were evaluated separately and graded between 1 and 8 (9). The PAS results were divided into 3 categories: no penetration or aspiration (PAS 1), penetration (PAS 2-5), and aspiration (PAS 6-8). A team including a physical medicine and rehabilitation specialist and a swallowing therapist assessed the videofluoroscopic swallowing study.

The FOIS was used to determine the patient's functional oral intake status at 7 levels; level 1 to 3 indicate varying degrees of non-oral feeding (tube dependency); level 4 or 5 indicates total oral intake with special preparations, and level 6 or 7 indicate total oral intake without special preparations (11).

\section{Treatment protocol}

All patients received an 8-week swallowing rehabilitation as part of an inpatient rehabilitation program that was a standard procedure after brain injury. The therapy was planned 3 days a week and each session was completed in $45 \mathrm{~min}$. The following order was followed in line with the patient's needs and videofluoroscopic study results:

1. Bolus size and consistency modifications,

2. Postural maintenance (informing the patient and family/ caregiver about the importance of optimal posture in terms of swallowing function), 
3. Compensatory strategies (e.g., chin tuck, head tilt),

4. Exercise (e.g., range of motion of the lips, jaw, tongue, hyolaryngeal mobilization, chewing training, thermal tactile stimulation),

5. Swallowing maneuver (e.g., effortful swallow, Mendelsohn maneuver).

\section{Statistical Analysis}

All statistical tests were performed using Statistical Package for the Social Sciences Statistics for Mac version 20.0 (Armonk, NY: IBM Corp., 2011). The distributions of continuous variables were determined using a Kolmogorov-Smirnov test. Nominal data were displayed as the frequency and percentage. Continuous data were reported as mean \pm standard deviation, median and interquartile range where appropriate. Categorical data were compared with the chi-square test. Within-group comparisons were performed using a paired t-test or Wilcoxon signed-rank test as appropriate. $p<0.05$ was considered statistically significant.

\section{Results}

The study included 271 patients [mean age: $50.0 \pm 20.1$ years; male sex: $179(66.1 \%)$; stroke: 175 (64.6\%), TBI: 58 (21.4\%); ABI: 13 (4.8\%); glioma: 18 $(6.6 \%)$ and encephalitis: 7 (2.6\%)]. The demographic characteristics of the patients are shown in Table 1. Patients with stroke were older [age, median: 61.0 (50.071.0) years], and the patients with TBI were younger [age, median: 24.0 (15.0-38.0) years] compared with the other patients with other etiologies. Patients with $A B I$ and encephalitis seemed to have more risk of aspiration pneumonia compared to stroke patients. Sixty-three of the patients had a history of aspiration pneumonia, while $28.6 \%(n=18)$ of them were tube dependent, and $30.2 \%(n=19)$ showed improvement in FOIS outcomes after the rehabilitation program. Encephalitis was the leading etiology associated with a history of aspiration pneumonia (57.1\%) and tracheostomy (71.4\%). Patients with $\mathrm{ABI}$ had the highest rate of tracheostomy history (84.6\%), and more than half of the patients with $\mathrm{ABI}$ had experienced aspiration pneumonia (53.8\%). Although $35.6 \%(n=31)$ of the patients had normal feeding, $33.3 \%(n=29)$ of 87 patients with a history of tracheostomy use were tube dependent.

Table 2 displays changes in FOIS level of the patients after a rehabilitation program compared to baseline. Significant improvement was observed on the FOIS in patients with stroke (pretreatment: $5.9 \pm 1.9$

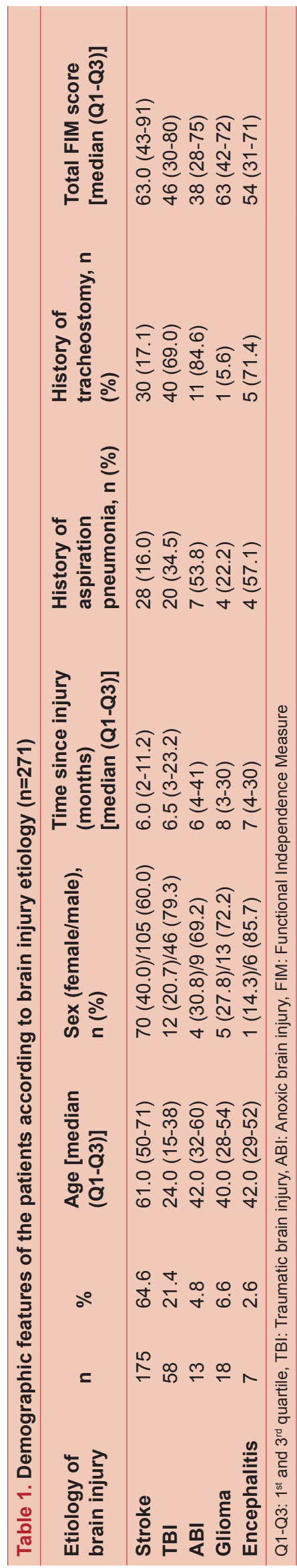


vs. posttreatment: $6.1 \pm 1.9, \mathrm{p}=0.011$ ) and TBI (pretreatment: $5.3 \pm 2.4$ vs. posttreatment: 5.9 $\pm 7.0, p=0.007$ ). However, there were no significant changes in the FOIS in patients with $\mathrm{ABI}$ (pretreatment: $6.3 \pm 1.4$ vs. posttreatment: $6.5 \pm 1.4$, $p=0.317$ ), glioma (pretreatment: $4.5 \pm 2.9$ vs. posttreatment: $5.0 \pm 2.7, p=0180$, and encephalitis (pretreatment: $3.4 \pm 3.0$ vs. posttreatment: $6.0 \pm 2.2, p=0.083$ ).

Comparison of the patients who had improvement in FOIS and those who did not were compared. Patients who showed an improvement were significantly younger (43.0 \pm 19.9 years of age) than the patients who did not improve $(51.7 \pm 19.9$ years of age) $(p<0.05)$.

PAS scores of the patients are shown in Table 3. In the stroke and TBI groups, significant improvements were observed in all three consistencies (solid-pudding-liquid) after treatment. In the encephalitis group, a significant increase was observed only in the solid consistency after treatment.

A hundred seventy-five patients with stroke were grouped according to the artery involvement: 128 (73.1\%) with the middle cerebral artery (MCA), 27 (15.4\%) with the posterior cerebral artery (PCA), 14 (8.0\%) with the anterior cerebral artery (ACA), and $6(3.4 \%)$ with internal carotid artery (ICA) involvement. Patients with MCA and PCA syndromes showed statistically significant improvement on the FOIS; however, the patients with
ICA and ACA syndromes did not (Table 4). The only significant improvement on the PAS was observed in the liquid scores of patients with MCA syndrome (Table 5).

\section{Discussion}

In this study, data on the change in oral intake and swallowing dysfunction after swallowing therapy in patients with acquired brain damage in a tertiary rehabilitation center were examined. It was concluded that there were significant improvements in oral intake and aspiration-penetration rates in patients with stroke and TBI after swallowing therapy, but the changes in $\mathrm{ABI}$, glioma, and encephalitis groups were not significant. When the patients with stroke were examined according to their vascular involvement, it was determined that MCA and PCA syndromes gave a significant response to the treatment. The results are valuable because it examines the response to treatment of different groups of patients with neurological swallowing disorders and different vascular involvement.

Neurogenic dysphagia is a common cause of mortality in neurological disorders. However, most studies into neurogenic dysphagia have focused on patients with stroke (12-15). However, there is a paucity of evidence indicating the effectiveness the dysphagia rehabilitation in TBI. Most of the studies involving patients with TBI consist of heterogeneous patient groups $(16,17)$. However, patients with stroke and TBI have different

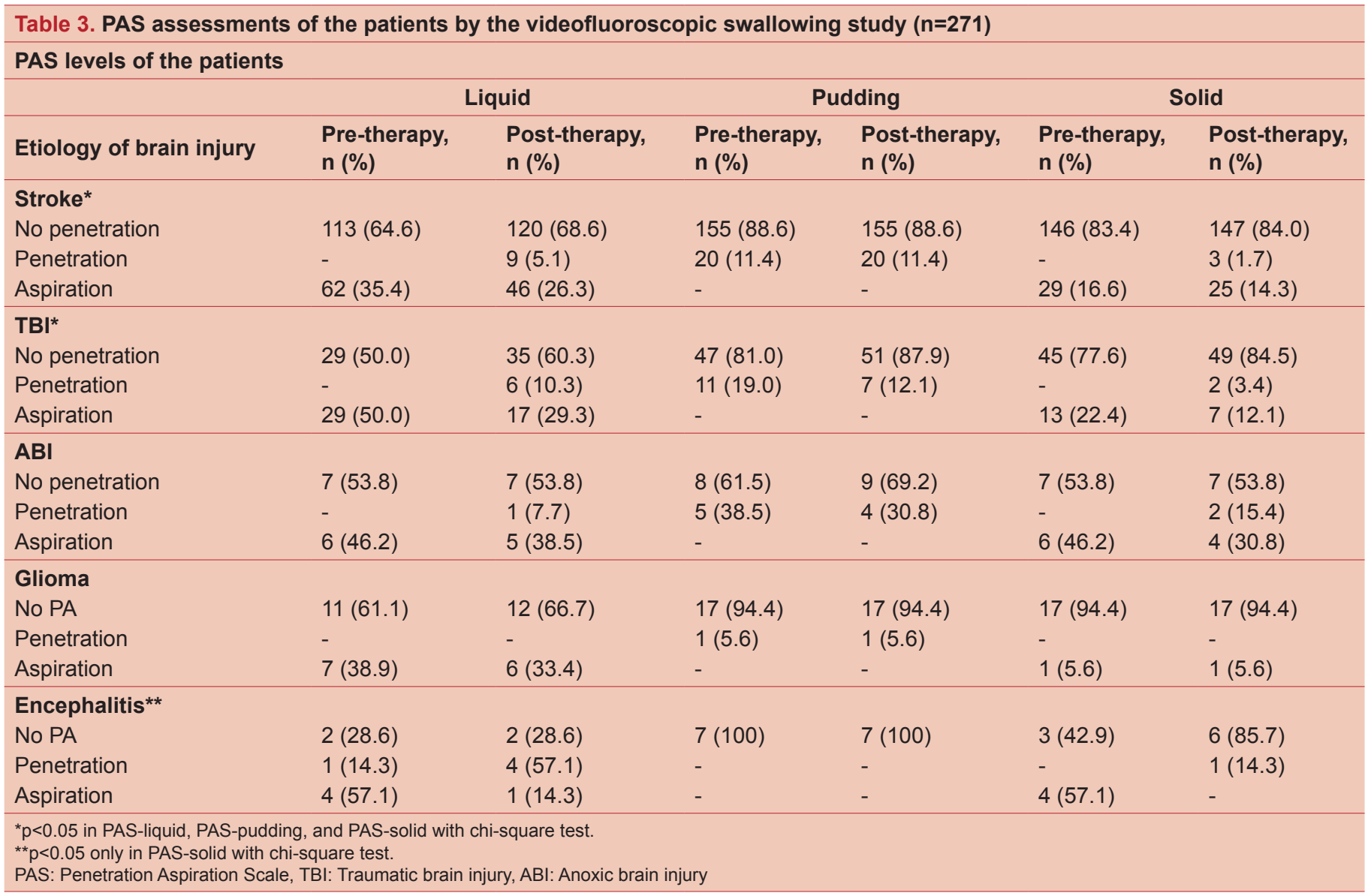


characteristics. Stroke survivors are seen at older ages than patients with TBI, which may explain the FIM scores similar to the others. While vascular damage due to stroke most often affects focal areas unilaterally, brain damage in TBI is usually bilateral and diffuse. Cognitive impairment is more common in patients with TBI (18). With regard to oropharyngeal damage, speech problems are more frequent in stroke patients, whereas delay in pharyngeal peristalsis is more frequent and severe in patients with TBI (19). In the present study, improvements were observed in the oral intake and penetration-aspirating rates of patients with TBI during dysphagia rehabilitation, similar to patients with stroke. According to our FOIS data, $27.6 \%$ of the patients with TBI were tube dependent, while only $14.6 \%$ of the patients with stroke were tube dependent. Improvement in functional oral intake was seen in $16.6 \%$ of the patients with stroke and $27.6 \%$ of the patients with TBI.
Dysphagia is observed in approximately $26 \%$ of patients with brain tumors (20). The most important factors determining the frequency and severity of dysphagia are tumor location and size (21). In the study where Park et al. (22) compared the dysphagia characteristics of patients with stroke and brain tumors, it was concluded that patients with brain tumors were not different from stroke patients in terms of age, lesion location, and degree of swallowing dysfunction. However, they also reported that whether the tumor is malignant or benign does not affect the degree of swallowing dysfunction. In a study comparing the functional gains in brain tumor and stroke patients after inpatient rehabilitation, the authors concluded that patients with brain tumors benefit from treatment similar to stroke patients (23). Similarly, in the study by Wesling et al. (21), in which changes in swallowing dysfunction after inpatient rehabilitation between the

Table 4. Improvement and comparison of the FOIS according to vascular area involvement in patients with stroke ( $\mathrm{n}=175$ )

\begin{tabular}{|c|c|c|c|c|c|c|c|c|c|c|}
\hline \multicolumn{2}{|c|}{$\begin{array}{l}\text { Vascular area } \\
\text { involvement }\end{array}$} & \multicolumn{2}{|c|}{$\begin{array}{l}\text { Tube dependent } \\
(1-3)\end{array}$} & \multicolumn{2}{|c|}{ Oral intake (4-5) } & \multicolumn{2}{|c|}{ Oral intake (6-7) } & \multicolumn{3}{|c|}{ Total FOIS changes } \\
\hline & n (\%) & $\begin{array}{l}\text { Pre- } \\
\text { therapy, } \\
\text { n (\%) }\end{array}$ & $\begin{array}{l}\text { Post- } \\
\text { therapy, } \\
\text { n (\%) }\end{array}$ & $\begin{array}{l}\text { Pre- } \\
\text { therapy, } \\
\text { n (\%) }\end{array}$ & $\begin{array}{l}\text { Post- } \\
\text { therapy, } \\
\text { n (\%) }\end{array}$ & $\begin{array}{l}\text { Pre- } \\
\text { therapy, } \\
\text { n (\%) }\end{array}$ & $\begin{array}{l}\text { Post- } \\
\text { therapy, } \\
\text { n (\%) }\end{array}$ & $\begin{array}{l}\text { Pre-therapy } \\
\text { mean } \pm S D \\
\text { [median } \\
(Q 1-Q 3)]\end{array}$ & $\begin{array}{l}\text { Post-therapy } \\
\text { mean } \pm S D \\
\text { [median } \\
(Q 1-Q 3)]\end{array}$ & $\mathbf{p}$ \\
\hline ICA & $6(3.4)$ & $1(16.7)$ & $1(16.7)$ & $2(33.3)$ & $2(33.3)$ & $3(50.0)$ & $3(50.0)$ & $4.5 \pm 2.7[6(1-7)]$ & $4.6 \pm 2.8[6(1-7)]$ & 0.317 \\
\hline PCA & $27(15.4)$ & $8(29.6)$ & $2(7.4)$ & $4(14.8)$ & $5(18.5)$ & $15(55.6)$ & $20(74.1)$ & $5.2 \pm 2.6[7(1-7)]$ & $5.4 \pm 2.6[7(1-7)]$ & 0.046 \\
\hline
\end{tabular}

FOIS: Functional oral intake status. ICA: Internal carotis artery, MCA: Middle cerebral artery, ACA: Anterior cerebral artery, PCA: Posterior cerebral artery, TBI: Traumatic brain injury, ABI: Anoxic brain injury

Table 5. PAS assessments of the patients with stroke according to vascular area involvement $(n=175)$ PAS

\begin{tabular}{|c|c|c|c|c|c|c|}
\hline & \multicolumn{6}{|l|}{ PAS } \\
\hline & \multicolumn{2}{|c|}{ Liquid } & \multicolumn{2}{|c|}{ Pudding } & \multicolumn{2}{|c|}{ Solid } \\
\hline & Pre-therapy, n (\%) & $\begin{array}{l}\text { Post-therapy, n } \\
(\%)\end{array}$ & $\begin{array}{l}\text { Pre-therapy, n } \\
\text { (\%) }\end{array}$ & $\begin{array}{l}\text { Post-therapy, n } \\
(\%)\end{array}$ & $\begin{array}{l}\text { Pre-therapy, } \\
\text { n (\%) }\end{array}$ & $\begin{array}{l}\text { Post-therapy, } \\
\text { n (\%) }\end{array}$ \\
\hline \multicolumn{7}{|l|}{ ICA } \\
\hline No penetration & $2(33.3)$ & $2(33.3)$ & $4(66.7)$ & $4(66.7)$ & $3(50.0)$ & $4(66.7)$ \\
\hline Penetration & - & - & $2(33.3)$ & $2(33.3)$ & - & - \\
\hline \multicolumn{7}{|l|}{ MCA* $^{*}$} \\
\hline No penetration & $89(69.5)$ & $100(78.2)$ & $120(93.8)$ & $120(93.8)$ & $112(87.5)$ & $115(89.8)$ \\
\hline Penetration & - & - & $8(6.3)$ & $8(6.3)$ & - & - \\
\hline Aspiration & $39(30.5)$ & $28(21.8)$ & - & - & $16(12.5)$ & $13(10.2)$ \\
\hline \multicolumn{7}{|l|}{ ACA } \\
\hline No penetration & $16(59.3)$ & $20(74.1)$ & $21(77.8)$ & $21(77.8)$ & $21(77.8)$ & $21(77.8)$ \\
\hline Penetration & - & - & $6(22.2)$ & $6(22.2)$ & - & - \\
\hline Aspiration & $11(40.7)$ & $7(25.9)$ & - & - & $6(22.2)$ & $6(22.2)$ \\
\hline
\end{tabular}

ICA 
two groups were analyzed, no difference was found between the two groups. In the present study, contrasting with the previous studies, desired gains could not be obtained after treatment in patients with brain tumors. The reason for this finding may be that the cognitive functions of the patients in our study were worse, or the lesion location and size were different compared to other studies.

Dysphagia is common in patients with encephalitis, especially in the presence of brainstem involvement (24). Dysphagia causes both deteriorations in quality of life and vital complications such as aspiration pneumonia. There are not enough studies on the importance of swallowing therapy for treating dysphagia in patients with encephalitis (25). In this study, only the change in PAS-fluid values was found to be significant after swallowing therapy.

Although there are different types of dysphagia treatment in the literature, swallowing rehabilitation is an effective method for patients with neurogenic dysphagia. Since swallowing has a complex physiology, only a single exercise or maneuver is not sufficient to rehabilitate the swallowing dysfunction (26). For this, it is necessary to understand patients' swallowing dysfunction correctly and to determine appropriate strategies. In the present study, swallowing therapy (3 days a week, 8 weeks, 24 sessions) was implemented in line with the patient's needs and video-fluoroscopic study results. Bolus size and consistency modifications, postural maintenance, compensatory strategies (e.g., chin tuck, head tilt), exercises (e.g., range of motion of the lips, jaw, tongue, hyolaryngeal mobilization, chewing training), and swallowing maneuver (effortful swallow, Mendelsohn maneuver) were applied in treatment. Our outcomes showed that the tube dependency and oral feeding restrictions decreased after therapy in all patients, but improvement rates in patients with stroke and TBI were most pronounced.

Different central nervous system regions from the cerebral cortex to the medulla oblongata affect swallowing physiology. The cerebrum is responsible for cognition and coordination as well as oral chewing and peristalsis. The brain stem is responsible for pharyngeal swallowing, laryngeal elevation, glottic closure, and cricopharyngeal relaxation. Within the cortex, lesions of the insula, the frontal operculum, and the primary sensorimotor cortex are most commonly associated with swallowing dysfunction. Kim et al. (27) investigated the patterns of post-stroke swallowing difficulties according to the vascular territories involved in the stroke and found that territorial anterior infarcts are more related to oral phase dysfunction whereas territorial posterior infarcts are more related to pharyngeal dysfunction. In the present study when the patients with stroke were examined separately according to the vascular area involvement, videoflouroscopic examinations showed that aspiration levels were higher in the patients with ICA and ACA involvement compared to patients with MCA and PCA involvement. As expected, the aspiration of liquids was evident in all stroke patients. However, the most tolerable consistency was pudding for all post-stroke examinations. There was a significant gain after swallowing rehabilitation in patients with MCA or PCA involvement according to the functional oral outcomes due to the major artery of swallowing areas nourished by these vessels.

There were several limitations in this study. Firstly, the study was in a retrospective design and lacked a control group that did not receive swallowing therapy. Therefore, the potential influence of spontaneous recovery on results could not be measured. However, it is unethical to leave patients without treatment while swallowing treatment is available. Secondly, the number of patients with $\mathrm{ABI}$, encephalitis, and brain tumors was small compared with the group of stroke patients. Also, the lack of data on cognitive performance which is associated with the swallowing function is considered another limitation. Among the notable strengths are the large number of patients overall and the inclusion of different etiologies of brain injury.

\section{Conclusion}

Swallowing therapy is an important part of rehabilitation in patients with neurogenic dysphagia after ABI. To our knowledge, this is the first study among Turkish patients who showed the efficacy of swallowing therapy in different etiologies of ABI. The outcomes were overall better for patients with TBI and stroke, and the patients with stroke with MCA and PCA involvement gained more benefit from the treatment.

\section{Ethics}

Ethics Committee Approval: The study protocol was approved by the Local Ethics Committee (Gülhane Military Medical Academy, decision no: 1642-63250, date: 04.11.2014).

Informed Consent: Retrospective study.

Peer-review: Externally peer-reviewed.

\section{Authorship Contributions}

Concept: Ö.İ., Design: S.K., E.Y., Data Collection or Processing: R.A., Ö.I., Analysis or Interpretation: B.A., S.K., Literature Search: B.A., Ö.I., E.Y., Writing: B.A., S.K.

Conflict of Interest: No conflict of interest was declared by the authors.

Financial Disclosure: The authors declared that this study received no financial support

\section{References}

1. Bakheit AM. Management of neurogenic dysphagia. Postgrad Med J. 2001;77:694-699.

2. Ertekin C, Aydogdu I, Yüceyar N, et al. Electrodiagnostic methods for neurogenic dysphagia. Electroencephalogr Clin Neurophysiol. 1998;109:331-340. 
3. Wirth R, Dziewas R. [Neurogenic dysphagia]. Internist (Berl). 2017;58:132-140.

4. Black-Schaffer RM, Kirsteins AE, Harvey RL. Stroke rehabilitation. 2. Co-morbidities and complications. Arch Phys Med Rehabil. 1999;80(5 Suppl 1):8-16.

5. No authors listed. Consensus conference. Rehabilitation of persons with traumatic brain injury. NIH Consensus Development Panel on Rehabilitation of Persons With Traumatic Brain Injury JAMA. 1999;282:974-983.

6. Mackay LE, Morgan AS, Bernstein BA. Swallowing disorders in severe brain injury: risk factors affecting return to oral intake. Arch Phys Med Rehabil. 1999;80:365-371.

7. Palmer JB, Drennan JC, Baba M. Evaluation and treatment of swallowing impairments. Am Fam Physician. 2000;61:24532462.

8. Küçükdeveci AA, Yavuzer G, Elhan AH, Sonel B, Tennant A. Adaptation of the Functional Independence Measure for use in Turkey. Clin Rehabil. 2001;15:309-311.

9. Luchesi KF, Campos BM, Mituuti CT. Identification of swallowing disorders: the perception of patients with neurodegenerative diseases. Codas. 2018;30:e20180027.

10. Karaduman A, Serel S, Ünlüer Ö, Demir N. The Penetration Aspiration Scale: an interrater reliability study. Turkish Journal of Physiotherapy and Rehabilitation. 2012;23:151-155.

11. Crary MA, Mann GD, Groher ME. Initial psychometric assessment of a functional oral intake scale for dysphagia in stroke patients. Arch Phys Med Rehabil. 2005;86:1516-1520.

12. Bülow M, Speyer R, Baijens L, Woisard V, Ekberg $O$ Neuromuscular electrical stimulation in stroke patients with oral and pharyngeal dysfunction. Dysphagia. 2008;23:302309.

13. Kushner DS, Peters K, Eroglu ST, Perless-Carroll M, JohnsonGreene D. Neuromuscular electrical stimulation efficacy in acute stroke feeding tube-dependent dysphagia during inpatient rehabilitation. Am J Phys Med Rehabil. 2013;92:486495.

14. Carnaby G, Hankey GJ, Pizzi J. Behavioural intervention for dysphagia in acute stroke: a randomised controlled trial. Lancet Neurol. 2006;5:31-37.

15. Park JW, Kim Y, Oh JC, Lee HJ. Effortful swallowing training combined with electrical stimulation in post-stroke dysphagia: a randomised controlled study. Dysphagia. 2012;27:521-527.
16. Sdravou K, Walshe M, Dagdilelis L. Effects of carbonated liquids on oropharyngeal swallowing measures in people with neurogenic dysphagia. Dysphagia. 2012;27:240-250.

17. Ludlow CL, Humbert I, Saxon K, Poletto C, Sonies B, Crujido L. Effects of surface electrical stimulation both at rest and during swallowing in chronic pharyngeal Dysphagia. Dysphagia. 2007;22:1-10.

18. Howle AA, Baguley IJ, Brown L. Management of Dysphagia Following Traumatic Brain Injury. Curr Phys Med Rehabil Rep. 2014;2:219-230.

19. Lazarus C, Logemann JA. Swallowing disorders in closed head trauma patients. Arch Phys Med Rehabil.1987;68:79-84.

20. Mukand JA, Blackinton DD, Crincoli MG, Lee JJ, Santos BB. Incidence of neurologic deficits and rehabilitation of patients with brain tumors. Am J Phys Med Rehabil. 2001;80:346-350.

21. Wesling $M$, Brady $S$, Jensen $M$, Nickell $M$, Statkus $D$, Escobar N. Dysphagia outcomes in patients with brain tumors undergoing inpatient rehabilitation. Dysphagia. 2003;18:203210.

22. Park DH, Chun MH, Lee SJ, Song YB. Comparison of swallowing functions between brain tumor and stroke patients. Ann Rehabil Med. 2013;37:633-641.

23. O'Dell MW, Barr K, Spanier D, Warnick RE. Functional outcome of inpatient rehabilitation in persons with brain tumors. Arch Phys Med Rehabil. 1998;79:1530-1534.

24. Dietrich-Burns K, Lewis WJ, Lesley DY, Solomon NP. Silent aspiration and recovery from dysphagia in a case of bickerstaff brainstem encephalitis. Mil Med. 2013;178:121-124.

25. Barikroo A, Lam PM. Comparing the effects of rehabilitation swallowing therapy vs. functional neuromuscular electrical stimulation therapy in an encephalitis patient: a case study. Dysphagia. 2011;26:418-423.

26. Rogus-Pulia N, Robbins J. Approaches to the rehabilitation of dysphagia in acute poststroke patients. Semin Speech Lang. 2013;34:154-169.

27. Kim SY, Kim TU, Hyun JK, Lee SJ. Differences in videofluoroscopic swallowing study findings according to the vascular territory involved in stroke. Dysphagia. 2014;29:444449. 